\title{
Propriedades farmacológicas da Aloe vera (L.) Burm. f.
}

FREITAS, V.S. ${ }^{*}$; RODRIGUES, R.A.F. ${ }^{2,3}$; GASPI, F.O.G. ${ }^{2}$

${ }^{1}$ Pós-graduação do Curso de Especialização de Fitoterapia da Fundação Hermínio Ometto - UNIARARAS, Av. Dr. Maximiliano Baruto 500 - Araras, SP, CEP: 13607-339. ${ }^{2}$ Docentes do Curso de Especialização de Fitoterapia da Fundação Hermínio Ometto - UNIARARAS. ${ }^{3}$ Centro Pluridisciplinar de Pesquisas Químicas Biológicas e Agrícolas - UNICAMP. *autora correspondente: ve_freitas@hotmail.com

\begin{abstract}
RESUMO: A Aloe vera (L.) Burm.f. tem sido utilizada há milhares de anos na medicina tradicional para o tratamento de diversos males. $O$ intuito desse trabalho foi o levantamento bibliográfico de artigos que evidenciassem a atividade farmacológica da Aloe vera. A revisão contemplou livros e periódicos nacionais e internacionais indexados nas bases de dados MEDLINE, LILACS e SciElo, nos idiomas português, inglês e espanhol, utilizando as palavras-chave citadas. Após o levantamento bibliográfico, constatou-se que várias atividades biológicas são atribuídas a Aloe vera. Evidências sugerem eficácia no tratamento da psoríase, herpes genital, queimaduras e hiperglicemia. Além disto, também foram demonstradas atividades antineoplásica, antimicrobiana, anti-inflamatória e imunomodulatória por estudos in vitro e in vivo, entretanto, na cicatrização de feridas, os resultados foram conflitantes. No tratamento de dermatite por radiação e em queimaduras solares sua eficácia não foi comprovada e foram relatados casos de hepatite aguda devido ao consumo de preparações orais. Tendo em vista as várias atividades comprovadas e poucos relatos acerca de sua contra indicação, conclui-se que o uso desta espécie corrobora o vasto uso popular.
\end{abstract}

Palavras-chave: Aloe vera, babosa, fitoterapia, plantas medicinais.

\begin{abstract}
Pharmacological activities of Aloe vera (L.) Burm. f. The Aloe vera (L.) Burm.f. has been used for thousands of years in traditional medicine to treat various ailments. The aim of this study was to carry out a bibliographical review on the pharmacological activity of Aloe vera. This review included books and national and international journals indexed to MEDLINE, LILACS and SciELO, in Portuguese, English and Spanish, using the key words mentioned. After the literature review, we found that several biological activities have been attributed to Aloe vera. Evidence suggests efficacy in the treatment of psoriasis, genital herpes, burns and hyperglycemia. Moreover, antineoplastic, antimicrobial, anti-inflammatory and immunomodulatory activities have also been demonstrated by in vitro and in vivo studies; however, in wound healing, the results were conflicting. In the treatment of radiation dermatitis and sunburn, its efficacy has not been proven, and cases of acute hepatitis from the consumption of oral preparations have been reported. Considering the various proven activities and the few reports about the contraindications of Aloe vera, we conclude that the use of this species confirms its wide popular usage.
\end{abstract}

Keywords: Aloe vera, herbal medicine, medicinal plants.

\section{INTRODUÇÃO}

\section{História da Aloe vera}

A história da Aloe vera é antiga e se encontra presente na literatura de diversas culturas. Seu nome provavelmente se origina da palavra arábica alloeh, que significa substância amarga e brilhante. O primeiro registro do uso da $A$. vera foi feito em uma tabuleta de argila da Mesopotâmia datada de 2100 a.C. (Atherton, 1997).

Conhecida no Egito antigo como a "planta da imortalidade", teria sido usada por Cleópatra nos cuidados da pele e do cabelo. Menções existentes na Bíblia, de acordo com muitos historiadores, seriam na verdade de outras plantas, a Excoecaria agalocha L. ou da Aquillaria agalocha L. (Teske \& Trentini, 1997; Alonso, 2007). 
É citada na enciclopédia História Natural de Plínio, o Velho (23 - 79 d.C.) e na Matéria Médica de Dioscórides, considerado o fundador da Farmacognosia, que fez referência ao cheiro forte e gosto amargo da $A$. vera, e ao seu uso no tratamento de irritações da pele e na cura de furúnculos e feridas (Haller, 1990).

Foi trazida por comerciantes para o mercado londrino em 1693 e em 1843 quantias consideráveis eram importadas. Atualmente é plantada em grande escala em diversos países, como México, EUA e China. Foi reconhecida pela Farmacopeia Britânica como droga oficial em 1932 sendo aceita também em diversas outras farmacopeias. É muito comum no Brasil onde é popularmente utilizada na cicatrização de feridas, no tratamento de queimaduras, conjuntivite, dores reumáticas dentre outros males (Haller, 1990; Alonso, 2007; Lorenzi \& Matos, 2008; Guerra et al., 2008).

\section{Descrição Botânica}

A Aloe vera $(L)$ Burm. f. pertence à família Aloaceae que inclui cerca de 15 gêneros e 800 espécies. É uma planta herbácea que cresce em qualquer tipo de solo, mas é melhor adaptada aos leves e arenosos e não exige muita água. Suas folhas são verdes, grossas, suculentas e medem de 30 a 60 centímetros de comprimento. Suas flores são vistosas, apresentam tonalidade brancoamarelada, em formato tubular. Na literatura é encontrada com as sinonímias Aloe barbadensis Mill., Aloe barbadensis var. chinensis Haw., Aloe perfoliata var. vera L., Aloe chinensis Bak. e Aloe vera var. chinensis Berger. Popularmente é chamada de babosa, aloe, aloe-de-barbados e aloe-decuraçao. (Lorenzi \& Matos, 2008; WHO, 1999).

\section{Partes utilizadas na terapêutica}

A Aloe vera demora de quatro a cinco anos para atingir a maturidade e suas folhas podem ser divididas em duas partes. Da parte mais externa pode se extrair um suco, que quando concentrado e seco recebe a denominação de Aloé. Esse suco flui espontaneamente das folhas cortadas e possui cor marrom escura, além de forte odor e sabor muito amargo. É composto principalmente por derivados antracênicos sendo as aloínas (barbaloína e isobarbaloína) os mais conhecidos (Atherton, 1997; WHO, 1999).

Durante os séculos dezoito e dezenove o Aloé foi popularmente prescrito por seus efeitos catárticos, que são mais fortes que de qualquer outra planta conhecida. Atualmente, não é mais recomendado como primeira escolha entre as preparações laxativas devido aos efeitos colaterais resultantes, tais como cólica severa e náusea (Haller, 1990; WHO, 1999; Cunha, 2005).
Após a eliminação dos tecidos mais externos da folha, obtêm-se um gel mucilaginoso com aparência viscosa e incolor que recebe o nome de gel de $A$. vera. Constitui-se principalmente por água e polissacarídeos, além de 70 outros componentes, tais como, vitamina A,B,C e E, cálcio, potássio, magnésio e zinco, diversos aminoácidos, enzimas e carboidratos. (Teske \& Trentini, 1997; Femenia et al., 1999; Carvalho, 2005; Surjushe, 2008).

Alterações sazonais e de cultivo podem afetar a composição do gel. Os níveis de polissacarídeos, um dos componentes ativos, por exemplo, são menores nas plantas bem irrigadas. O processamento das folhas também deve ser feito logo após a colheita, pois o gel oxida rapidamente quando entra em contato com o ar (Yaron, 1993; Rodríguez-González et al., 2011).

O gel é muito utilizado como matéria prima na indústria cosmética, alimentícia e farmacêutica e diversas técnicas são empregadas para sua conservação (Atherton, 1997; Eshun \& He, 2004; Cunha, 2005;).

A pasteurização é provavelmente a técnica de processamento mais utilizada pela indústria e um dos métodos sugeridos é o HTST (temperatura alta, tempo curto, sigla em inglês) onde o gel é aquecido à $65^{\circ} \mathrm{C}$ por no máximo 15 minutos seguido por rápido resfriamento à $5^{\circ} \mathrm{C}$ ou menos. Rodríguez-González et al. (2011) relatam diminuição significativa nos níveis de acemanana, um dos bioativos do gel, além de outras alterações em amostras pasteurizadas a $85^{\circ} \mathrm{C}$, provavelmente devido à degradação térmica.

Outro método utilizado é a desidratação, que de acordo com Femenia et al. (2003) provocou perda significativas de acemanana e outros compostos quando as amostras foram tratadas com temperaturas acima dos $60^{\circ} \mathrm{C}$.

O emprego de técnicas de processamento que conservem os ativos do gel de maneira adequada é essencial para que o mesmo apresente os efeitos farmacológicos esperados (Eshun \& He, 2004).

\section{RESULTADOS}

\section{Atividade anti-inflamatória e cicatrizante}

Estudos feitos em animais ou por meio de testes in vitro identificaram algumas substâncias como sendo parcialmente responsáveis pela atividade anti-inflamatória e cicatrizante da $A$. vera e vários mecanismos foram propostos para explicar sua influência nesses processos.

A acemanana, polissacarídeo encontrado em grande quantidade no gel de Aloe vera, foi capaz de estimular in vitro macrófagos murinos da 
linhagem RAW 264.7 a liberarem interleucina-6, fator de necrose tumoral- $\alpha$ e óxido nítrico. A combinação entre a acemanana e interferon- $\gamma$ potencializou esses efeitos, sugerindo, portanto uma ação sinérgica (Zhang \& Tizard, 1996).

Em outro experimento in vitro, a acemanana, nas concentrações de 2 até $16 \mathrm{mg} / \mathrm{mL}$, aumentou de maneira significativa a proliferação de fibroblastos gengivais e estimulou a secreção do fator de crescimento de queratinócitos - 1 (KGF-1) e do fator de crescimento vascular endotelial (VEGF), além de colágeno do tipo I. Todas essas substâncias estão diretamente ligadas com a cicatrização, uma vez que desempenham papeis importantes, como re-epitelização tecidual, formação de vasos sanguíneos e formação de tecido conjuntivo (Jettanacheawchankit et al., 2009).

Davis et al. (1994) relataram que a manose6-fosfato, polissacarídeo presente no gel da A. vera, acelerou o processo de cicatrização e diminuiu a inflamação em camundongos na dosagem de 300 $\mathrm{mg} / \mathrm{kg}$. Foram feitos ensaios de cicatrização de ferida e também de indução de edema de orelha com óleo de cróton. Os autores sugerem, como possível mecanismo de ação, a ligação da manose-6-fosfato em receptor presente em fibroblastos, contribuindo dessa forma com o processo de cicatrização.

Outros ensaios de cicatrização de ferida foram feitos em ratos por Jettanacheawchankit et al., 2009 e por Takzare et al. 2009. Ambos observaram resposta estatisticamente significante na cicatrização das feridas tratadas com Aloe vera quando comparados com grupo controle, com redução do tamanho da ferida após sete dias de tratamento.

Proteínas e glicoproteínas isoladas do gel de $A$. vera também exibiram atividade antiinflamatória in vitro ao reduzirem de maneira significativa as enzimas COX -2 e lipoxigenase de maneira comparável a anti-inflamatórios não esteroidáis como diclofenaco e nimesulida. Quando testadas em animais foram capazes de acelerar a cicatrização e aumentar a proliferação celular (Choi et al., 2001; Das et al., 2011).

A aloína e a aloe-emodina foram testadas in vitro por Park et al. (2009) e seu efeito antiinflamatório foi comparável com o da quercetina e campeferol.

Em humanos, os resultados sobre o efeito cicatrizante da $A$. vera são conflitantes. Schmidt \& Greenspoon (1991) relataram que o uso do gel de $A$. vera aumentou o tempo de cicatrização em feridas cirúrgicas quando comparado com tratamento idêntico que não incluía o gel.

No entanto, Eshghi et al. (2010) publicaram recentemente um estudo randomizado, duplo cego, placebo controlado, onde pacientes que passaram por cirurgia de hemorroidectomia aberta e que fizeram aplicação do creme contendo $A$. vera apresentaram menor dor pós-operatória, melhora na cicatrização e menor consumo de analgésicos quando comparado com o grupo placebo.

Esses resultados conflitantes podem ser parcialmente explicados se levarmos em conta o caráter hidrofílico dos componentes ativos presentes na $A$. vera, e que estes atravessam com muita dificuldade as barreiras impostas pela pele. Sabe-se que a combinação do gel de $A$. vera com ultrassom, microcorrente ou na forma de lipossomas pode acelerar a cicatrização e diminuir a inflamação ao favorecer a penetração dos ativos no tecido (Takahashi et al., 2009; Mendonça et al., 2009; Maia-Filho et al., 2011).

No tratamento de queimaduras a $A$. vera foi testada em estudo publicado por Khorasani et al. (2009) que fizeram comparação entre a sulfadiazina de prata a $1 \%$ (tratamento comumente utilizado) e um creme contendo $A$. vera a $0,5 \%$ em 30 indivíduos com queimadura de segundo grau. $\mathrm{O}$ creme contendo $A$. vera se mostrou superior ao promover a cicatrização e reepitelização da pele em menos de 16 dias, enquanto os ferimentos tratados com o creme contendo sulfadiazina de prata levaram em média 19 dias para cicatrização.

No caso de queimaduras solares a mesma eficácia não pôde ser comprovada. De acordo com trabalho randomizado e duplo cego publicado por Puvabanditsin \& Vongtongsri (2005) o creme contendo $70 \%$ de $A$. vera não exerceu efeito protetor ou auxiliou no tratamento de queimadura solar em 20 voluntários testados.

\section{Atividade antineoplásica}

Estudos experimentais relatam a atividade antineoplásica da $A$. vera frente a diversas linhagens de câncer. Supõe-se que a aloína, aloe-emodina e a acemanana sejam parcialmente responsáveis por essa atividade.

Vários são os mecanismos sugeridos para o efeito citotóxico provocado pela $A$. vera, e esses parecem depender da dose utilizada e do tipo de tumor. Uma das hipóteses levantadas é a de que há uma redução na proporção de células na fase mitótica por indução de apoptose provocada pelas antraquinonas (Esmat et al. 2006).

Perturbações no ciclo e na diferenciação celular, estimulação do sistema imune, além de marcante atividade antioxidante também são sugeridas, o que resultaria em efeito antiproliferativo. Estudo recente, utilizando uma solução feita com mel de abelhas e $A$. vera em ratos, demonstrou diminuição progressiva do tamanho do tumor quando comparado com grupo controle (Niciforovic et al., 2007, Tomasin \& Gomes-Marcondes, 2010).

Rev. Bras. Pl. Med., Campinas, v.16, n.2, p.299-307, 2014. 
Em outro estudo a utilização de $A$. vera tópica e/ou oralmente em ratos também resultou em uma diminuição no número de tumores além de aumento do tempo do aparecimento dos mesmos (Saini et al., 2010).

Lissoni et al. (2009) publicaram estudo preliminar com outra espécie do gênero Aloe, a Aloe arborescens, combinada à quimioterapia em 240 pacientes com câncer em fase de metástase. Foi possível evidenciar uma maior taxa de sobrevivência e de regressão do tumor, além de alívio da fadiga e astenia, quando comparados ao grupo controle. Outro estudo preliminar, feito com pacientes com câncer em estágio avançado demonstrou maior estabilização da doença e aumento do tempo de sobrevivência no grupo tratado com a combinação da A. vera e do hormônio melatonina (Lissoni et al., 1998).

Os resultados disponíveis até o momento, apesar de escassos, são encorajadores e estudos clínicos bem conduzidos e com maior número de pacientes ainda são necessários para verificar sua real efetividade.

\section{No tratamento da psoríase}

No tratamento da psoríase a $A$. vera teve sua eficácia clínica demonstrada em um estudo randomizado, duplo cego, placebo controlado de Syed et al. (1996a) que contaram com 60 pacientes que sofriam de psoríase de grau leve à moderado. Os pacientes foram divididos em grupo controle e placebo e aplicaram nas lesões três vezes ao dia um creme hidrofílico contendo extrato de $A$. vera a $0,5 \%$ ou um creme similar sem o ativo. No final das quatro semanas de tratamento $83 \%$ dos pacientes que fizeram uso do creme com $A$. vera foram considerados curados contra somente $6 \%$ do grupo placebo. O tratamento com o creme de A. vera foi bem tolerado, sem efeitos adversos e os pacientes puderam notar excelente melhora ou completa resolução das lesões.

Choonhakarn et al. (2010) compararam, em estudo randomizado e duplo cego a eficácia do creme de $A$. vera com um creme contendo $0,1 \%$ de triancinolona acetonida em oitenta pacientes portadores de psoríase de estágio leve a moderado. Observaram que o creme contento $A$. vera foi mais eficaz na melhora dos sintomas clínicos do que o tratamento convencional, no entanto, ambos foram similares na melhora da qualidade de vida do paciente.

\section{radiação}

No tratamento da dermatite e mucosite por

O primeiro relato do uso da $A$. vera para o tratamento de dermatite causada por radiação foi feito por Collins \& Collins (1935). A paciente que sofria de severa dermatite no lado esquerdo da face foi orientada a aplicar topicamente o gel da folha fresca na área afetada. Vinte e quatro horas depois, a mesma relatou melhora na coceira e na sensação de queimação e cerca de um mês após o uso diário do gel houve completa cura. Outro relato foi feito por Loveman (1936), onde dois pacientes com dermatite e ulcerações severas nas mãos causadas por radiação, foram tratados com o gel da folha fresca da $A$. vera por períodos de tempo variados. Ambos pacientes obtiveram cicatrização completa da dermatite e das úlceras.

Devido a esses relatos a $A$. vera tem sido indicada no tratamento de dermatite por radiação, apesar de não haver protocolo de tratamento estabelecido.

Dois estudos clínicos de fase III foram feitos em pacientes com câncer tratados com radioterapia. Em ambos, não foi possível evidenciar efetividade do gel de $A$. vera no alívio da dermatite (Williams, 1996; Merchant, 2007).

Segundo Su et al. (2004) a A. vera não apresentou benefício estatisticamente significante na melhora do grau da mucosite ou na tolerância a radioterapia quando comparada ao grupo controle, no tratamento da mucosite provocada por radiação, em estudo duplo cego, randomizado, placebo controlado, feito em 58 pacientes portadores de câncer de cabeça e pescoço. Já Puataweepong et al. (2009) conduziram estudo semelhante ao feito por Su et al. e relataram que a incidência de mucosite severa foi menor no grupo tratado com suco de $A$. vera do que no grupo controle. Tal fato pode ser explicado, de acordo com os autores, pelo preparo do suco com o gel fresco das folhas da $A$. vera sobre condições bem controladas, preservando assim um maior número de substâncias ativas.

\section{No tratamento da hiperglicemia e dislipidemia}

A atividade hipoglicemiante da $A$. vera foi verificada em animais por meio de estudos como o realizado por Tanaka et al. (2006). Uma linhagem de ratos portadores de distúrbios metabólicos similares aos causados pelo Diabetes tipo 2, como hiperglicemia, obesidade e resistência insulínica foram tratados via oral com $25 \mu \mathrm{g} /$ animal/dia de gel de A. vera ( nas concentrações de 20,30 e 50 mg/ $\mathrm{mL}$ ) e $1 \mathrm{ug} / \mathrm{animal} / \mathrm{dia}$ de 5 diferentes fitoesteróis isolados desta espécie (lofenol, 24-metil-lofenol, 24-etil-lofenol, cicloartanol e 24-metileno-cliartanol). Os autores relataram que o grupo teste, após 33 dias de tratamento, obteve redução significativa ( $p<0,05$ para as concentrações de 20 e $30 \mathrm{mg} /$ $\mathrm{mL}$ e $\mathrm{p}<0,005$ na dose de $50 \mathrm{mg} / \mathrm{mL}$ ) nos níveis de glicose em jejum e também não perderam peso, sintoma apresentado pelos animais controles, já

Rev. Bras. PI. Med., Campinas, v.16, n.2, p.299-307, 2014. 
que estes, devido a alta taxa de glicemia perdem grande quantidade de glicose na urina.

Outros estudos também foram feitos em ratos com Diabetes tipo 1 induzida por estreptozotocina. Os autores puderam verificar atividade antioxidante, redução da glicose plasmática a níveis normais, além disso, exames histológicos feitos no pâncreas, fígado, rim e intestinos puderam demonstrar efeito protetor do gel de $A$. vera quando comparada ao grupo controle (Rajasekaran et al., 2005; Noor et al., 2008).

Em humanos, Ngo et al. (2010) publicaram recentemente artigo de revisão sobre a utilização da $A$. vera para o tratamento do Diabetes e da dislipidemia. Os autores verificaram a existência de 8 estudos clínicos com 5285 pacientes no total e chegaram a conclusão de que há indícios de benefício do uso da $A$. vera na redução da glicose e do colesterol.

\section{Outros usos descritos na literatura}

A utilização de xarope contendo gel de $A$. vera na concentração de $50 \%$ proporcionou melhora clínica e de indicadores respiratórios funcionais em pacientes portadores de asma brônquica, sem que houvesse efeitos colaterais significativos. Os pacientes do grupo controle reduziram a utilização de outros medicamentos antiasmáticos e pôde-se demonstrar também que a nebulização do extrato de $A$. vera protegeu o paciente frente a hiperreatividade bronquial provocada pela carbacolina (Rivas et al., 2004).

A Aloe vera parece ser eficaz também no tratamento da conjuntivite, de acordo com estudo descritivo exploratório publicado por Guerra et al. (2008) onde vinte e seis pacientes relataram controle da dor, diminuição da vermelhidão e cura após 3 dias de tratamento ao fazer uso do gel de uso oftalmológico.

No tratamento do líquen plano bucal, a aplicação tópica da $A$. vera promoveu melhora na qualidade de vida dos pacientes, apesar de não ter apresentado diferença significativa na diminuição da dor quando comparado com placebo (SalazarSanchez et al., 2010). Em outro estudo randomizado, duplo-cego, placebo-controlado Choonhakarn et al. (2008) relatam que o gel de $A$. vera provocou melhora clinica e sintomatológica do líquen plano bucal quando comparado a grupo placebo.

No líquen plano vulvar a aplicação local do gel de $A$. vera resultou na melhora das lesões em $82 \%$ do grupo tratado comparado a $4 \%$ do grupo placebo (Rajar et al., 2008).

Em estudo randomizado, duplo-cego, placebo-controlado com a utilização de solução oral contendo gel de $A$. vera foi possível verificar melhora clínica e histológica em pacientes com colite ulcerativa ativa de maneira mais frequente do que quando comparado a grupo controle (Langmead et al. 2004).

A $A$. vera possui amplo espectro antimicrobiano atuando em fungos, vírus e em bactérias Gram positivas e Gram negativas. Estudos identificaram alguns compostos com ação bactericida ou bacteriostática em Staphylococcus aureus, Pseudomonas aeruginosa, Escherichia coli, Helicobacter pylori, Salmonella typhi, Bacillus cereus, Candida albicans, Shigella flexneri, Streptococcus pyogenes, Mycobacterium tuberculosis entre outros. O pirocatecol, ácido cinâmico, ácido ascórbico e ácido p-cumárico são alguns dos compostos identificados que provavelmente atuam de forma sinérgica (Puerto et al., 2001; Ferro et al. 2003; Tamura et al., 2009; Lawrence et al., 2009; Gupta et al. 2010; Das et al., 2011).

A atividade da $A$. vera frente ao Herpes genital foi verificada em dois estudos duplo cego, placebo controlados. Os estudos contaram com cerca de 180 pacientes no total e o creme hidrofílico contendo $0,5 \%$ de extrato de $A$. vera foi o que apresentou maior taxa de cura quando comparado ao gel de $A$. vera ou com o placebo (Syed et al. 1996b; Syed et al. 1997).

Uma solução oral preparada com gel de $A$. vera aumentou em mais de três vezes a biodisponibilidade das vitaminas $\mathrm{C}$ e $\mathrm{E}$ em testes feitos em humanos normais, abrindo margem para sua utilização em suplementos vitamínicos (Vinson et al., 2005).

Outro estudo relata a diminuição de rugas e aumento de expressão de pró-colágeno tipo 1 em um grupo de mulheres acima de 45 anos que fizeram ingestão oral de solução feita de gel de $A$. vera em pó dissolvido em água destilada. Esses resultados, no entanto, são limitados já que não houve grupo controle (Cho et al. 2009).

Devido a seu poder hidratante a indústria de cosméticos e higiene pessoal faz amplo uso do gel de $A$. vera em diversos tipos de formulações, como cremes, xampus e sabonetes. Em alguns países é incorporado a cremes de barbear com o objetivo de auxiliar na cicatrização dos cortes (Eshun $\& \mathrm{He}, 2004)$. De acordo com estudo publicado por Dal'Belo et al. (2006) o poder hidratante do gel de $A$. vera sobre a pele se deve provavelmente a um mecanismo umectante. A aplicação da formulação contendo o gel aumentou de maneira significativa o teor de água do estrato córneo, sem provocar oclusão ou alterações nas barreiras da pele.

O gel desta planta é adicionado também em diversos cremes dentais sob a alegação de possuir efeito antiplaca e antigengivite, apesar da falta de dados na literatura que confirmem essa informação. Com o objetivo de verificar a eficácia 
da $A$. vera nesses casos, Oliveira et al. (2008) testaram em 30 pacientes os efeitos antiplaca e antigengivite de creme comercial contendo $A$. vera e não observaram diferenças significativas quando comparado a grupo controle. Já com a utilização de enxaguatório bucal na concentração de $50 \%$, Villalobos et al. (2008) observaram diminuição da placa e da gengivite quando comparado a grupo placebo. Esses resultados discrepantes podem ser talvez explicados pela maior concentração da A. vera presente no enxaguatório bucal. Outro estudo conduzido por Lee et al. (2004) verificaram por meio de testes in vitro efeito inibitório de creme dental comercial contendo gel de $A$. vera sobre $A$. viscosus, $C$. albicans, S. mutans e $S$. sanguis, que são alguns dos microorganismos mais envolvidos com doenças bucais. Os resultados desse estudo, porém, podem ter sofrido interferência de outras substâncias com atividade antimicrobiana presentes no creme dental analisado.

\section{Contra indicações e efeitos colaterais}

Devido à presença de antraquinonas, não é recomendada sua utilização oral durante a gravidez, já que seu efeito estimulatório no intestino grosso pode provocar reflexos na musculatura uterina induzindo aborto. As antraquinonas também causam, quando em excesso, forte diarréia, cólicas, náusea e consequentemente perda de eletrólitos o que resulta em disfunção cardíaca e neuromuscular, principalmente se o paciente já fizer uso de glicosídeos cardíacos, diuréticos ou anti-arrítmicos (Baretta et al., 2009; WHO, 1999).

O uso crônico também pode resultar em lesão do aparelho neuromusuclar e formação de um "cólon de laxantes", além de provocar lesões renais crônicas (Wagner \& Wiesenauer, 2006).

Atualmente constam na literatura casos de hepatite aguda causada pelo consumo oral de A. vera. O primeiro relato foi feito na Alemanha, por Rabe et al. (2005) que reportaram o caso de uma mulher de 57 anos, que após ingerir tabletes com $500 \mathrm{mg}$ de extrato de $A$. vera durante quatro semanas apresentou sintomas como icterícia, prurido e dor abdominal. Por meio de exames laboratoriais foi possível verificar níveis anormais de alanina aminotransferase (1480 U/L - normal: <22 $\mathrm{U} / \mathrm{L})$, aspartato aminotransferase (711 U/L - normal: $<15$ U/L) e a biópsia hepática revelou hepatite aguda severa. Uma semana após a suspensão dos tabletes a paciente se tornou assintomática e os níveis de alanina aminotransferase lentamente retornaram ao normal.

Em 2007, Bottenberg et al. relatou caso similar ao de Rabe et al. onde uma paciente de 73 anos queixou-se de náusea, fadiga, perda de peso dentre outros sintomas. Exames laboratoriais e biópsia hepática indicaram hepatite aguda e somente após ser questionada especificadamente sobre o uso de medicamentos a base de plantas medicinais a mesma admitiu que nos últimos 5 anos vinha consumindo cápsulas contendo $500 \mathrm{mg}$ de $A$. vera a cada 2 ou 3 dias para tratar de constipação. Níveis de bilirrubina, alanina aminotransferase e aspartato aminotransferase voltaram ao normal após alguns meses de suspensão das cápsulas.

Mais recentemente, outros três casos foram relatados por Yang et al. (2010) envolvendo o consumo de preparações contendo $A$. vera e alterações hepáticas tóxicas.

De maneira interessante, Chandan et al. (2007) relatam efeito hepatoprotetor de extrato aquoso de $A$. vera frente a danos causados por tetracloreto de carbono em ratos. Os níveis de alanina aminotransferase, aspartato aminotransferase, bilirrubina e de outros parâmetros bioquímicos analisados foram restaurados sem que houvesse sinais de toxicidade em doses de até $2 \mathrm{~g} / \mathrm{kg}$ rato. Os autores sugerem que esse efeito hepatoprotetor do extrato aquoso se deva a presença de antioxidantes que evitam a depleção da glutationa e protegem contra a peroxidação lipidica. Ozsoy et al. (2009) identificaram a presença de diversos antioxidantes naturais, como, flavonóides, ácido ascórbico, $\beta$-caroteno e $\alpha$-tocoferol, em extrato aquoso da folha da $A$. vera. Atividade antioxidante do extrato aquoso não foi observada quando se utilizou o gel da folha.

De acordo com estudo publicado por Holanda et al. (2009) o consumo oral de extrato de $A$. vera provocou maior captação de pertecnetato de sódio, um radiofármaco utilizado em diagnóstico por imagens, no sangue, fêmur, rins, fígado, estômago, testículos e tireóide em ratos testados, o que pode resultar em diagnósticos incorretos ou má interpretação de resultados por imagem. $O$ estudo também relatou aumento dos níveis de alanina aminotransferase e aspartato aminotransferase e diminuição de colesterol, glicose e triglicerídeos quando comparado a grupo controle.

Há relatos na literatura de dermatite de contato e sensação de queimação provocadas pelo uso tópico de gel da $A$. vera. Essas reações provavelmente se devem a presença de resíduos de antraquinonas no gel utilizado (Horgan, 1988; WHO, 1999).

A comercialização de sucos ou outros alimentos contendo Aloe vera foi proibida recentemente pela ANVISA (2011) devido a falta de evidências científicas que comprovassem sua segurança e em virtude de relatos de reações adversas. 


\section{CONCLUSÃO}

Numerosas atividades biológicas foram atribuídas a Aloe vera ao longo dos anos, e isso provavelmente se deve a combinação dos diversos ativos existentes em sua composição. Oresultado da pesquisa bibliográfica revelou que esta espécie é eficaz no tratamento da psoríase, herpes genital, hiperglicemia e em queimaduras. As atividades antineoplásica, antimicrobiana, anti-inflamatória e imunomodulatória também foram demonstradas por estudos in vitro e in vivo.

$\mathrm{Na}$ cicatrização de feridas os resultados são conflitantes, e isso pode estar relacionado ao caráter hidrofílico dos componentes ativos que enfrentam dificuldade de atravessar as barreiras impostas pela pele (Takahashi, 2009). Também pode ser devido às diferenças na forma de extração dos princípios ativos ou nos métodos de armazenagem utilizados pelos pesquisadores.

No tratamento de dermatite por radiação, em queimaduras solares ou como protetor solar, o gel da planta não demonstrou eficácia.

Em relação à sua toxicidade, foram relatados casos de hepatite aguda e também foram observados alguns sintomas tais como, cólicas, náuseas e diarréia com o consumo de preparações orais contendo $A$. vera.

É imprescindível a padronização do uso da $A$. vera, com objetivo de conservar melhor suas características químicas e a definição da melhor forma de sua aplicação. Fica evidente a enorme gama de atividades que esta espécie possui, sendo necessários, porém, estudos controlados com maior número de participantes, para que seja definida dose e forma segura para o seu uso.

\section{REFERÊNCIA}

ALONSO, J. Tratados de fitofarmacos y nutraceuticos. 2.ed. Argentina: Corpus Editorial, 2007. 1150p.

ANVISA. Informe Técnico $\mathbf{n . 4 7}$, de 16 de novembro de 2011. Esclarecimentos sobre comercialização de Aloe vera (babosa) e suas avaliações de segurança realizadas na área de alimentos da Anvisa. Disponível em: http://www.anvisa.gov.br. Acesso em: 21 de nov. de 2011.

ATHERTON, P. Aloe vera revisited. The British Journal of Phytotherapy, v.4, n.4, p.176-83, 1997.

BARETTA, Z. et al. Aloe-induced hypokalemia in a patient with breast cancer during chemotherapy. Annals of Oncology, v.20, n.8, p.1445-46, 2009.

BOTTENBERG, M.M. et al. Oral aloe vera induced hepatitis. The Annals of Pharmacotherapy, v.41, n.10, p.1740-43, 2007.

CARVALHO, J.C.T. Formulário Médico Farmacêutico de Fitoterapia. 2.ed. Editora Pharmabooks, 2005. 404p.

CHANDAN, B.K. et al. Hepatoprotective potential of Aloe barbadensis Mill. against carbon tetrachloride induced hepatotoxicity. Journal of Ethnopharmacology, v.111, n.3, p. 560-66, 2007.

$\mathrm{CHO}, \mathrm{S}$. Dietary Aloe vera supplementation improves facial wrinkles and elasticity and it increases the type 1 procollagen gene expression in human skin in vivo. Annals of Dermatology, v.21, n.1, p.6-11, 2009.

CHOI, C.W. et al. The wound-healing effect of a glycoprotein fraction isolated from aloe vera. British Journal of Dermatology, v.145, n.4, p.535-45, 2001.

CHOONHAKARN, C. et al. A prospective, randomized clinical trial comparing topical aloe vera with $0,1 \%$ triamcinolone acetonide in mild to moderate plaque psoriais. Journal of the European Academy of Dermatology and Venereology, v.24, n.2, p.168-172, 2010.

CHOONHAKARN, C. et al. The efficacy of aloe vera gel in the treatment of oral lichen planus: a randomized controlled trial. The British Journal of Dermatology, v.158, n.3, p.573-77, 2008.

COLLINS, C.E.; COLLINS, C. Roentgen dermatitis treated with fresh whole leaf of aloe vera. American Journal of Roentgenology, v.33, p.396-97, 1935.

CUNHA, A.P. Farmacognosia e Fotoquímica. 1. ed. Lisboa: Fundação Calouste Gulbenkian, 2005. 670 p.

DAL'BELO, S.E. et al. Moisturizing effect of cosmetic formulations containing Aloe vera extract in different concentrations assessed by sking bioengineering techniques. Skin Research and Technology, v.12, n.4, p.241-46, 2006.

DAS, S. et al. Isolation and characterization of novel protein with anti-fungal and anti-inflammatory properties from Aloe vera leaf gel. International Journal of Biological Macromolecules, v.48, n.1, p.38-43, 2011.

DAVIS, R.H. et al. Anti-inflammatory and wound healing activity of a growth substance in Aloe vera. Journal of the American Podiatric Medical Association, v.84, n.2, p.77-81, 1994.

ESHGHI, F. et al. Effects of Aloe vera cream on posthemorrhoidectomy pain and wound healing: results of a randomized, blind, placebo-control study. The Journal of Alternative and Complementary Medicine, v.16, n.6, p.647-50, 2010.

ESHUN, K.; HE, Q. Aloe vera: A valuable ingredient for the food, pharmaceutical and cosmetic industries - a review. Critical Reviews in Food Science and Nutrition, v.44, n.2, p.91-96, 2004.

ESMAT, A.Y. et al. Cytotoxicity of a natural anthraquinone (aloin) agains human breast cancer cell lines with and without Erb-2-Topoisomerase Ila coamplification. Cancer Biology \& Therapy, v.5, n.1, p.97-103, 2006.

FEMENIA, A. et al. Compositional features of polysaccharides from Aloe vera (Aloe barbadensis Miller) plant tissues. Carbohydrate Polymers, v.39, n.2, p.109-117, 1999.

FEMENIA, A. et al. Effects of heat treatment and dehydration on bioactive polysaccharide acemannan and cell wall polymers from Aloe Barbadensis Miller. Carbohydrate Polymers, v.51, n.4, p.397-405, 2003.

FERRO, V.A. et al. In vitro susceptibilities of Shigella flexneri and streptococcus pyogenes to inner gel of Aloe barbadensis Miller. Antimicrobial Agents and Chemothererapy, v.47, n.3, p.1137-39, 2003.

GUERRA, M.F.L. et al. Uso empírico in natura de Aloe sp 
em portadores de conjuntivite. Revista de Enfermagem da UFPE, v.2, n.1, p. 36-46, 2008.

GUPTA, R. et al. Anti-tuberculosis activity of selected medicinal plants agains multi-drug resistant Mycobacterium tuberculosis isolates. Indian Journal of Medical Research, v.131, jun, p.809-13, 2010.

HALLER, J.S. A drug for all seasons Medical and Pharmacological history of Aloe. Bulletin of the New York Academy of Medicine, v.66, n.6, p.647-59, 1990. HOLANDA, C.M.C.X. et al. Effect of an extract of Aloe vera on the biodistribution of sodium pertechnetate (Na99mTcO4) in rats. Acta. Cirúrgica Brasileira, v.24, n.5, p. 383-86, 2009.

HORGAN, D.J. Widespread dermatitis after topical treatment of chronic leg ulcers and stasis dermatitis. Canadian Medical Association Journal, v.138, n.4, p.336-38, 1988.

JETTANACHEAWCHANKIT, $S$. et al. Acemannan stimulates gingival fibroblast proliferation; expressions of keratinocyte growth factor-1, vascular endothelial growth factor, and type 1 collagen; and wound healing. Journal of Pharmacological Sciences, v.109, p.525-31, 2009.

KHORASANI, G. et al. Aloe versus silver sulfadiazine creams for second-degree burns: a randomized controlled study. Surgery Today, v.39, n.7, p.587-591, 2009.

LANGMEAD, L. et al. Randomized, double-blind, placebocontrolled trial of oral aloe vera gel for active ulcerative colitis. Alimentary Pharmacology \& Therapeutics, v.19, n.7, p.739-47, 2004.

LAWRENCE, R. et al. Isolation, purification and evaluation of antibacterial agents from Aloe vera. Brazilian Journal of Microbiology, v.40, n.4, p.906-15, 2009.

LEE, S.S. et al. The antimicrobial potential of 14 natural herbal dentifrices: results of an in vitro diffusion method study. Journal of the American Dental Association, v.135, n.5, p.1133-1141, 2004.

LISSONI, P. et al. Biotherapy with the pineal immunomodulating hormone melatonin versus melatonin plus Aloe vera in untreatable advanced solid neoplasms. Natural Immunity, v.16, n.1, p.27-33, 1998.

LISSONI, P. et al. A Randomized study of chemotherapy versus biochemotherapy with chemotherapy plus Aloe arborescens in patients with metastatic cancer. In vivo, v.23, n.1, p.171-75, 2009.

LORENZI, H.; MATOS, F.J.A. Plantas medicinais no Brasil - Nativas e exóticas. 2.ed. São Paulo: Instituto Plantarum, 2008. 244p.

LOVEMAN, A.B. Leaf of aloe vera in treatment of roentgen ray ulcers. Archives of Dermatology and Syphilology, v.36, p.838-43, 1937.

MAIA-FILHO, A.L.M. et al. Efeito do gel da babosa (Aloe barbadensis Mill.) associado ao ultrassom em processo inflamatório agudo. Revista Brasileira de Plantas Medicinais, v.13, n.2, p.146-50, 2011.

MENDONÇA, F.A.S. et al. Effects of the application of Aloe vera (L.) and microcurrent on the healing of wounds surgically induced in wistar rats. Acta Cirúrgica Brasileira, v.24, n.2, p.150-55, 2009.

MERCHANT, T.E. et al. A phase III trial comparing an anionic phospholipid-based cream and aloe verabased gel in the prevention of radiation dermatitis in pediatric patients. Radiation Oncology, v.2, n.44,
2007. Disponível em: <http://www.ro-journal.com/ content/2/1/45>. Acesso em: 25 abr. 2011.

NGO, M.G. et al. Oral Aloe vera for treatment of diabetes mellitus and dyslipidemia. American Journal of HealthSystem Pharmacy, v.67, n.21, p.1804-11, 2010.

NICIFOROVIC, A. et al. Antitumor effects of a natural anthracycline analog (aloin) involve altered activity of antioxidant enzymes in HeLaS3 cells. Cancer Biology \& Therapy, v.6, n.8, p.1211-16, 2007.

NOOR, A. et al. Antidiabetic activity of Aloe vera and histology of organs in streptozotocin-induced diabetic rats. Current Science, v.94, n.8, p.1070-76, 2008.

OLIVEIRA, S.M.A. et al. Effect of a dentifrice containing Aloe vera on plaque and gengivitis control. A doubleblind clinical study in humans. Journal of Oral Applied Science, v.16, n.4, p.293-96, 2008.

OZSOY, N. et al. Implications for degenerative disorders - Antioxidative activity, total phenols, flavonoids, ascorbic acid, $\beta$-carotene and $\alpha$-tocopherol in Aloe vera. Oxidative Medicine and Cellular Longevity, v.2, n.2, p. 99-106, 2009.

PARK, M.Y. et al. Evaluation of aloin and aloe-emodin as anti-inflammatory agents in aloe by using murine macrophages. Bioscience, Biotechnology and Biochemistry, v.73, n.4, p.828-32, 2009.

PUATAWEEPONG, P. et al. The efficacy of oral Aloe vera juice for radiation induced mucositis in head and neck cancer patients: a double-blind placebo-controlled study. Asian Biomedicine, v.3, n.4, p.375-82, 2009.

PUERTO, E.C. et al. Efecto antiviral del extracto acuoso de Aloe barbadensis contra el vírus de la hepatitis $B$. Revista Cubana de Plantas Medicinales, v.6, n.1, p.7-11, 2001.

PUVABANDITSIN, P.; VONGTONGSRI, R. Efficacy of Aloe vera cream in prevention and treatment of sunburn and suntan. Journal of Medical Association of Thailand, v.88, n.4, p.173-76, 2005.

RABE, C. et al. Acute hepatitis induced by an Aloe vera preparation: a case report. World Journal of Gastroenterology, v.11, n.2, p.303-04, 2005.

RAJAR, U.D.M. et al. Efficacy of Aloe vera gel in the treatment of vulval lichen planus. Journal of the College of Physicians and Surgeons Pakistan, v.18, n.10, p.612-14, 2008.

RAJASEKARAN, S. et al. Antioxidant effect of Aloe vera gel extract in streptozotocin-induced diabetes in rats. Pharmacological Reports, v.57, n.1, p.90-96, 2005.

RIVAS, M.R. et al. Acción antiasmática del Aloe vera em pacientes. Revista Cubana de Plantas Medicinales, v.9, n.1, 2004.

RODRÍGUEZ-GONZALES, V.M. et al. Effects of pasteurization on bioactive polysaccharide acemannan and cell wall polymers from Aloe barbadensis Miller. Carbohydrate Polymers, v.86, n.4, p.1675-83, 2011.

SAINI, M. et al. Anti-tumor activity of Aloe vera against DMBA/croton oil-induced skin papillomagenesis in Swiss albino mice. Journal of Environmental Pathology, Toxicology and Oncology, v.29, n.2, p.127-35, 2010.

SALAZAR-SÁNCHEZ, N. et al. Efficacy of topical Aloe vera in patients with oral lichen planus: a randomized double-blind study. Journal of Oral Pathology and Medicine, v.39, n.10, p.735-40, 2010.

SCHMIDT, J.M.; GREENSPOON, J.S. Aloe vera dermal 
wound gel is associated with a delay in wound healing. Obstetrics \& Gynecology, v.78, n.1, p.115-17, 1991.

SU, C.K. et al. Phase II double-blind randomized study comparing oral aloe vera versus placebo to prevent radiation-related mucositis in patients with head-andneck neoplasms. International Journal of Radiation Oncology, Biology, Physics, v.60, n.1, p.171-77, 2004.

SURJUSHE, A. et al. Aloe vera: A short review. Indian Journal of Dermatology, v.53, n.4, p.163-66, 2008.

SYED, T.A. et al. Management of psoriais with Aloe vera extract in a hydrophilic cream: a placebocontrolled, double-blind study. Tropical Medicine and International Health, v.1, n.4, p.505-09, 1996a.

SYED, T.A. et al. Aloe vera extract $0,5 \%$ in a hydrophilic cream versus Aloe vera gel for the management of genital herpes in males. A placebo-controlled, doubleblind, comparative study. Journal of the European Academy of Dermatology and Venereology, v.7, n.3, p.294-95, 1996b.

SYED, T.A. et al. Management of genital herpes in men with $0,5 \%$ Aloe vera extract in a hydrophilic cream: a placebo-controlled double-blind study. Journal of Dermatological Treatment, v.8, n.2, p.99-102, 1997.

TAKAHASHI, M. et al. Liposomes encapsulating Aloe vera leaf gel extract significantly enhance proliferation and collagen synthesis in human skin cell lines. Journal of Oleo Science, v.58, n.12, p.643-50, 2009.

TAKZARE, N. et al. Influence of Aloe vera gel on dermal wound healing process in rat. Toxicology Mechanisms and Methods, v.19, n.1, p.73-77, 2009.

TAMURA, N. et al. Inhibition of infectious diseases by components from Aloe vera. Bioscience Biotechnology and Biochemistry, v.73, n.4, p.950-53, 2009.

TANAKA, M. et al. Identification of five phytosterols from aloe vera gel as anti-diabetic compounds. Biological \&
Pharmaceutical Bulletin, v.29, n.7, p.1418-22, 2006.

TESKE, M.; TRENTINI, A.M.M. Herbarium - Compêndio de Fitoterapia. 3.ed. Curitiba: Herbarium Laboratório Botânico, 1997. 317p.

TOMASIN, R.; GOMES-MARCONDES, M.C.C. Oral administration of Aloe vera and honey reduces walker tumour growth by decreasing cell proliferation and increasing apoptosis in tumor tissue. Phytotherapy Research, v.25, n.4, p.619-23, 2011.

VILLALOBOS, O.J. et al. Efecto de un enjuague bucal compuesto de Aloe vera en la placa bacteriana e inflamación gingival. Acta. Odontológica Venezolana, v.39, n.2, p. 16-24, 2001.

VINSON, J. et al. Effect of Aloe vera preparations on the human bioavailability of vitamins $C$ and $E$. Phytomedicine, v.12, n.10, p.760-765, 2005.

WAGNER, H.; WIESENAUER, M. Fitoterapia: Fitofármacos, Farmacologia e Aplicações clínicas. 2.ed. Pharmabooks, 2006. 424p.

WHO, World Health Organization. WHO Monographs on selected medicinal plants, vol. 1. Geneva: WHO Publications. 1999.

WILLIAMS, M.S. et al. Phase III double-blind evaluation of an Aloe vera gel as a prophylatic agent for radiationinduced skin toxicity. International Journal Radiation Oncology Biology Physics, v.36, n.2, p.345-49, 1996.

YANG, H.N. et al. Aloe-induced toxic hepatitis. Journal of Korean Medical Science, v.25, n.3, p.492-95, 2010.

YARON, A. Characterization of Aloe vera gel before and after autodegradation and stabilization of the natural fresh gel. Phytotherapy Research, v.7, n.7, p.11-13, 1993.

ZHANG, L.; TIZARD, I.R. Activation of a mouse macrophage cell line by acemannan: The major carbohydrate fraction from Aloe vera gel. Immunopharmacology, v.35, p.119-28, 1996. 